\title{
Prenatal acetaminophen exposure and risk of wheeze at age 5 years in an urban low-income cohort
}

\author{
Matthew S Perzanowski, ${ }^{1,2}$ Rachel L Miller, ${ }^{1,2,3}$ Deliang Tang, ${ }^{1,2}$ David Ali, \\ Robin S Garfinkel, ${ }^{1,4}$ Ginger L Chew, ${ }^{1,2}$ Inge F Goldstein, ${ }^{1,5}$ Frederica P Perera, ${ }^{1,2}$ \\ R Graham Barr ${ }^{1,3,5}$
}

\section{See Editorial, p99}

- Supplementary tables are published online only at http:// thx.bmj.com/content/vol65/ issue2.

${ }^{1}$ Columbia Center for Children's Environmental Health (CCCEH), New York, USA ${ }^{2}$ Department of Environmental Health Sciences, Mailman School of Public Health, New York, USA ${ }^{3}$ Department of Medicine, Columbia University College of Physicians and Surgeons, New York, USA ${ }^{4}$ Data Coordinating Center, Mailman School of Public Health, New York, USA ${ }^{5}$ Department of Epidemiology, Mailman School of Public Health, Columbia University, New York, USA

\section{Correspondence to}

R Graham Barr, PH-9E 105, Columbia Presbyterian Medical Center, 630 West 168th Street, New York, NY 10032, USA; rgb9@columbia.edu

Received 11 June 2009 Accepted 22 September 2009 Published Online First 22 October 2009

\begin{abstract}
Background Acetaminophen has been associated with asthma and is in part metabolised via the glutathione pathway. Inner-city minority children have high asthma morbidity and a relatively high frequency of a minor allele variant in the glutathione $S$ transferase $P$ i gene (GSTP1). We hypothesised that prenatal acetaminophen exposure would predict wheeze at age 5 years in an inner-city minority cohort and examined whether this association was modified by common polymorphisms in genes related to the glutathione pathway.

Methods An ongoing population-based birth cohort study of Dominican Republic and African-American children in New York prospectively assessed the use of analgesics during pregnancy and current wheeze at age 5 years in 301 children. Genotyping was conducted for GST polymorphisms. Binomial regression was used to adjust for potential confounders including postnatal acetaminophen use.

Results $34 \%$ of mothers reported acetaminophen use during pregnancy and $27 \%$ of children had current wheeze at 5 years. Prenatal exposure to acetaminophen predicted current wheeze (multivariate relative risk 1.71; $95 \% \mathrm{Cl} 1.20$ to $2.42 ; p=0.003$ ), and the risk increased monotonically with increasing number of days of prenatal acetaminophen exposure ( $p$ trend $<0.001$ ). $68 \%$ of children had at least one copy of the GSTP1 minor allele (Val). The risk of wheeze was modified by GSTP1 (additive interaction $p=0.009$ ) and was observed only among children with the GSTP1 minor allele.
\end{abstract}

Conclusions Prenatal exposure to acetaminophen predicted wheeze at age 5 years in an inner-city minority cohort. The risk was modified by a functional polymorphism in GSTP1, suggesting a mechanism involving the glutathione pathway.

\section{INTRODUCTION}

Acetaminophen use among children in the USA has increased substantially since the early 1980s and has become increasingly common among women during pregnancy so that most women in the USA take acetaminophen during pregnancy. ${ }^{1} 2$ These increases coincided with a doubling of the prevalence of asthma among children in the USA between 1980 and 1995..$^{3}$ A recent worldwide study showed that acetaminophen use in the first year of life, retrospectively ascertained, was associated with wheeze at age $6-7$ years across 30 countries. ${ }^{4}$ Prenatal exposure to acetaminophen was associated with an increased risk of asthma and wheeze at age 4-7years in birth cohort studies in the UK, Denmark and Spain. ${ }^{5-7}$ Whether these findings apply to children in inner-city communities in the USA, which have a disproportionately high burden of asthma, ${ }^{8}$ is uncertain.

The Val105 functional polymorphism in glutathione S-transferase $P i$ (GSTP) is particularly common among Hispanics and African-Americans and has been associated with altered susceptibility to asthma and allergic responses with exposure to various air pollutants. ${ }^{9-14}$ The presence of the minor allele in this polymorphism results in significant alteration of its conjugation activity, ${ }^{15}$ and genes in the GSTP pathway are relevant to acetaminophen pharmacokinetics. ${ }^{16} 17$ Whether polymorphisms in genes for the GST pathway modify the association between acetaminophen and asthma is unknown.

We hypothesised that acetaminophen exposure during pregnancy would be associated with wheeze at age 5 years in a birth cohort of African-American and Dominican Republic children living in a lowincome urban community, and that these associations would be modified by the GSTP1 gene.

\section{METHODS}

\section{Study sample}

The Columbia Center for Children's Environmental Health (CCCEH) recruited expectant mothers selfreporting as being of African-American race or of Dominican Republic origin living in Northern Manhattan and the South Bronx from a prenatal clinic system to participate in a birth cohort study. ${ }^{18}{ }^{19}$ Exclusion criteria were a diagnosis of diabetes or HIV infection, self-report of illicit drug use, residency in New York City for $<1$ year, active smoking during pregnancy and age <18years. Between 1998 and 2006, 2844 mothers were screened during pregnancy, of whom 1442 met the initial inclusion criteria. The most common exclusion criteria were related to residency (18\%), active smoking (13\%), race/ethnicity (13\%), maternal age (9.4\%) and maternal illness (6.8\%). Of the eligible mothers, 725 children (50\%) were fully enrolled, which included a prenatal home visit and donation of blood sample at the time of birth (cord and/or maternal blood). The most common reasons for non-enrollment among mothers who met the inclusion criteria were refusal to participate, loss of contact during pregnancy and lack of notification at delivery. Children of 11 mothers who did not complete prenatal questionnaire items on analgesic use were excluded from the study. Of the remaining 714 children, information on wheeze was available for 675, 614, 497 and 301 at ages 1, 2, 3 and 5 years, which represents $96 \%, 90 \%, 83 \%$ and $69 \%$ of 
children who had reached those respective ages in this ongoing birth cohort.

\section{Analgesic use during pregnancy}

Use of acetaminophen, ibuprofen and aspirin during pregnancy was ascertained in the third trimester. Mothers were specifically queried on "Tylenol", "Advil/Motrin", "cough medicine", "cold medicine", "aspirin" and "other". For each medication the number of days of use in each trimester was recorded.

Ascertainment of current wheeze and asthma-related end points Questionnaires on the child's environmental exposures and health status were administered to the mother during study visits at ages 1, 2, 3 and 5 years. Less detailed health follow-up questionnaires were administered by telephone at 3, 6, 9, 15, 18, 21 and 30 months. A child was considered to have wheezed in years 1,2 and 3 if at least one episode of wheeze was reported during at least one of the interviews pertaining to that year of life.

At age 5 years, current wheeze was defined as wheeze in the previous 12 months according to the validated ISAAC questionnaire. ${ }^{20}$ Additional end points included persistent wheeze (wheeze early in life persisting to age 5), transient wheeze (wheeze early in life but not at age 5), medication use for wheeze or difficulty breathing, and visits to the emergency department for wheeze or difficulty breathing. ${ }^{21}$

\section{Seroatopy}

Serum was obtained from 223 children (74\%) at the 5-year visit. IgE antibodies against inhalant allergens from Dermatophagoides farinae, mouse, cockroach, cat and dog were measured by ImmunoCAP (Phadia, Uppsala, Sweden). Children with IgE antibodies $\geq 0.35 \mathrm{IU} / \mathrm{ml}$ against any of these allergens were considered seroatopic.

\section{Potential confounders}

Relevant variables that could potentially confound the association between prenatal acetaminophen and wheeze were selected based on previous findings from our cohort and others. ${ }^{19} 2223$ Race/ethnicity, maternal asthma, domestic environmental tobacco smoke (ETS), cat in the home, birth order of the child, birth weight of child, age of mother and domestic sightings of cockroaches and mice were self-reported. Maternal educational attainment and receipt of Medicaid were used as proxies for socioeconomic status. Assessment of psychosocial stressors included sudden attacks of fear or panic for the mother, drug dealer or gang activity in the neighbourhood, homelessness since the child's birth and a previously defined material hardship variable. ${ }^{24}$ Postnatal use by the child of acetaminophen, ibuprofen or aspirin at was assessed at 6 months and 1, 2 and 3 years.

\section{Genotyping}

DNA was isolated from peripheral blood mononuclear cells and polymorphisms in the GSTP1 (Ile105Val, rs1695) and GSTT2 (G149T, rs2719) were assessed with the use of the ABI 7500 System (Applied Biosystems (ABI), Foster City, California, USA) in the TaqMan genotyping assay with primers and probes obtained from ABI. GSTP1 primers were GSTP1-F CCTGGTGGACATGGTGAATGAC; GSTP1-R CAGATGCTCACATAGTTGGTGTAGA. GSTT2 primers were GSTT2-F CTGCACAGACAAGACACTCAGT; GSTT2-R CTGGTTATGTATGCTGCACCTGA. The deletions of GSTT1 and GSTM1 were assessed by Multiplex PCR system as described previously. ${ }^{25}$ Children who were homozygous $(+/+)$ or heterozygous $(-/+)$ for GSTM1 (or GSTT1) were classified as GSTM1 (GSTT1) positive, and those who were homozygous deleted $(-/-)$ were classified as GSTM1 (GSTT1) null. Modification of the association between acetaminophen and wheeze by the presence of the minor allele in GSTP1 (ie, Ile/Val or Val/Val) was evaluated based on (1) previous associations with asthma ${ }^{11} 13$ and (2) altered conjugation kinetics of toxicants related to asthma among individuals with this genotype. ${ }^{15}$

\section{Statistical analysis}

Current wheeze at age 5 years was defined a priori as the primary end point, given the greater prognostic significance of wheeze as a child ages ${ }^{21}$ and bias in physician-diagnosed asthma in young children. ${ }^{20}$ Prenatal acetaminophen exposure was categorised as none versus any and by approximate tertiles of use (ie, 1, 2-4 and $\geq 5$ days). Given the high prevalence of wheeze, we used binomial regression ${ }^{26}$ to estimate relative risks (RR) and $95 \%$ confidence intervals (CI) for wheeze at age 5 by categories of prenatal acetaminophen exposure before and after adjustment for relevant covariates from table 1 which were included in the final model based on sensitivity analyses and convention for analyses with paediatric wheeze as an outcome.

Four GST polymorphisms were proposed for testing for interaction in the association between prenatal acetaminophen and wheeze. The statistical significance of effect modification by genotype was assessed by estimating the relative excess risk due to interaction (RERI) and its $95 \%$ CI as described by Hosmer and Lemeshow using our fully adjusted model. ${ }^{27} 28$ Data were analysed using SAS Version 9.1 (SAS Institute, Cary, North Carolina, USA), SPSS Version 16.0 (SPSS, Chicago, Illinois, USA) and Excel (Microsoft, Redmond, Washington, USA). ${ }^{27}$

\section{RESULTS}

Compared with the 135 eligible mothers who did not participate when the child was age 5, the 301 (69\%) mothers whose children did participate were significantly less likely to be of Dominican ethnicity and had a lower frequency of reported headaches and abdominal cramps during pregnancy (see table E1 in the online supplement). Acetaminophen use during pregnancy was reported by $34 \%$ of the mothers. In contrast, use of ibuprofen $(2.9 \%)$, aspirin $(0.8 \%)$ and cough $(2.3 \%)$ and cold $(1.3 \%)$ remedies during pregnancy were all. Table 1 shows the characteristics of the mothers and the children stratified by acetaminophen use during pregnancy.

\section{Asthma symptoms and seroatopy at age $\mathbf{5}$ years}

Current wheeze was reported for $26.9 \%$ of the children at age 5 years. Prenatal acetaminophen exposure predicted current wheeze at age 5 years (RR 1.71 ; 95\% CI 1.20 to 2.42 ; $\mathrm{p}=0.003$ ) in analyses adjusting for sex, ethnicity, birth order, maternal asthma, maternal hardship, ETS exposure and postnatal acetaminophen use. Similar relationships in adjusted analyses were observed for other asthma outcomes at age 5 years including use of asthma medications (RR 1.75; 95\% CI 1.26 to 2.43; $\mathrm{p}<0.001$ ) and emergency department visits for wheeze or difficulty breathing (RR 2.08; 95\% CI 1.35 to 3.21 ; $<<0.001$ ). Prenatal acetaminophen exposure predicted persistent wheeze (RR 1.65; $95 \%$ CI 1.12 to $2.44 ; p=0.012$ ) but not transient wheeze ( $R R$ $0.88 ; 95 \%$ CI 0.62 to $1.25 ; p=0.49$ ). There were no statistically significant associations between child's postnatal use of acetaminophen at 6 months, 1, 2 or 3 years and wheeze at age 5 years $(\mathrm{p}=0.37,0.81,0.11$ and 0.46 , respectively).

The frequency of prenatal exposure to acetaminophen predicted current wheeze at 5 years monotonically (table 2). Additional adjustment for other covariates listed in table 1 including antibiotic and ibuprofen use during pregnancy did not appreciably alter this association. The frequency of prenatal exposure to acetaminophen also predicted seroatopy at age 5 years. 
Table 1 Characteristics of mothers at time of pregnancy and children at age $5(n=301)$

\begin{tabular}{|c|c|c|}
\hline \multirow[b]{2}{*}{ Maternal demographic data } & \multicolumn{2}{|c|}{$\begin{array}{l}\text { Acetaminophen use during } \\
\text { pregnancy }\end{array}$} \\
\hline & $\begin{array}{l}\text { No } \\
(n=198)\end{array}$ & $\begin{array}{l}\text { Yes } \\
(n=103)\end{array}$ \\
\hline Age, mean (SD), years & $24.7(4.8)$ & $25.1(5.5)$ \\
\hline $\begin{array}{l}\text { Race/ethnicity } \\
\text { African-American, } n(\%)\end{array}$ & $99 / 198(50)$ & $39 / 103(38)^{*}$ \\
\hline Dominican, n (\%) & $99 / 198(50)$ & $64 / 103(62)^{*}$ \\
\hline $\begin{array}{l}\text { Health } \\
\text { Self-reported asthma, } \mathrm{n}(\%)\end{array}$ & 48/198 (24) & $28 / 103(27)$ \\
\hline Reported severe headaches in pregnancy, $n(\%)$ & $39 / 191(20)$ & $36 / 98(37)^{*}$ \\
\hline Reported occasional headaches in pregnancy, $\mathrm{n}(\%)$ & $96 / 195(49)$ & $76 / 101(75)^{*}$ \\
\hline Reported abdominal cramps in pregnancy, $\mathrm{n}(\%)$ & $30 / 196(15)$ & $23 / 101(23)$ \\
\hline Reported vaginal infection in pregnancy, $\mathrm{n}(\%)$ & 24/191 (13) & $12 / 98(12)$ \\
\hline Attacks of sudden fear or panic $\neq, \mathrm{n}(\%)$ & $35 / 195(18)$ & $29 / 102(28)^{*}$ \\
\hline $\begin{array}{l}\text { Socioeconomic status } \\
\text { No high school degree, } n(\%)\end{array}$ & $71 / 194(37)$ & $37 / 102(36)$ \\
\hline Receiving Medicaid, n (\%) & 182/197 (92) & $90 / 103(87)$ \\
\hline$\dagger, n(\%)$ & $80 / 197(41)$ & $48 / 101(48)$ \\
\hline $\begin{array}{l}\text { Prenatal medication } \\
\text { Antibiotics, } \mathrm{n}(\%)\end{array}$ & $42 / 195(22)$ & $23 / 101(23)$ \\
\hline Ibuprofen, n (\%) & $5 / 198(2.5)$ & $5 / 103(4.9)$ \\
\hline Aspirin, $n(\%)$ & $2 / 198(1.0)$ & $0 / 103(0)$ \\
\hline Acetaminophen Trim & $0 / 198(0)$ & $43 / 99(43)$ \\
\hline Trimester 2, n (\%) & $0 / 198(0)$ & $46 / 99(47)$ \\
\hline Trimester, n (\%) & 0/198 (0) & 42/99 (42) \\
\hline \multicolumn{3}{|l|}{ Child demographic data } \\
\hline Male, n (\%) & $90 / 198(46)$ & $54 / 103(52)$ \\
\hline Ever lived in home with smoker, $\mathrm{n}(\%)$ & 93/198 (47) & $42 / 103(41)$ \\
\hline $\begin{array}{l}\text { Birth order } \\
\text { First born, } \mathrm{n}(\%)\end{array}$ & $100 / 198(51)$ & $48 / 103(47)$ \\
\hline Third or later born, $\mathrm{n}(\%)$ & $44 / 198(22)$ & $26 / 103(25)$ \\
\hline Ever lived in home with cat, $\mathrm{n}(\%)$ & $54 / 198(27)$ & $24 / 103(23)$ \\
\hline Domestic mice sightings common§, $\mathrm{n}(\%)$ & $50 / 190(26)$ & 29/102 (28) \\
\hline Domestic sightings of cockroaches common§, $n(\%)$ & $80 / 185(43)$ & $47 / 100(47)$ \\
\hline $\begin{array}{l}\text { Drug dealers or users seen on neighbourhood streets, } \mathrm{n} \\
(\%)\end{array}$ & $111 / 189(59)$ & $63 / 102(62)$ \\
\hline $\begin{array}{l}\text { Neighbourhood gang activity makes it difficult to feel } \\
\text { safe } \uparrow, n(\%)\end{array}$ & $43 / 189(23)$ & $30 / 102(29)$ \\
\hline Child ever homeless ${ }^{* *}, \mathrm{n}(\%)$ & $16 / 189(8.5)$ & $14 / 102(14)$ \\
\hline \multicolumn{3}{|c|}{$\begin{array}{l}{ }^{*} \mathrm{p}<0.05 \text { for the difference in the prevalence of demographic characteristics among children } \\
\text { with and without prenatal acetaminophen exposure. } \\
\text { tMother reported that in the past } 6 \text { months she and her family could not afford needed food, } \\
\text { rent, clothing or medical care or that gas/electricity was suspended because of bill } \\
\text { non-payment. } \\
\text { †Report of sudden attacks of fear or panic sometimes, often or very often. } \\
\text { SReported pest sightings more than never or rarely. } \\
\text { \Reported dangerous activity in the neighbourhood sometimes, often or very often. } \\
{ }^{* *} \text { Mother reported she had ever been homeless (slept outside, in a car or in a homeless } \\
\text { shelter) since the child was born. }\end{array}$} \\
\hline
\end{tabular}

The use of acetaminophen was similar across the first, second and third trimesters $(14.5 \%, 15.5 \%$ and $14.1 \%$, respectively). Exposure in the second trimester predicted current wheeze at age 5 years (adjusted RR 1.54; 95\% CI 1.06 to $2.25 ; \mathrm{p}=0.02$ ), as did exposure in the third trimester (RR $1.55 ; 95 \%$ CI 1.03 to 2.34; $p=0.04)$. The association with acetaminophen exposure in the first trimester was of similar magnitude but was not statistically significant (RR 1.41; 95\% CI 0.93 to $2.14 ; \mathrm{p}=0.11$ ).

There was no evidence for effect modification of the association by race/ethnicity, maternal asthma, maternal age, sex or ETS exposure (RERI: $p=0.60, p=0.41, p=0.42, p=0.60, p=0.36$, respectively). The association between prenatal exposure to acetaminophen and wheeze was of a larger magnitude in children without seroatopy (adjusted RR 4.24; 95\% CI 2.07 to 8.72 ; $\mathrm{p}<0.001)$ than in those with seroatopy (RR 1.63 ; $95 \%$ CI 0.93 to
2.86; $\mathrm{p}=0.084)$; however, the interaction term was not significant (RERI: $\mathrm{p}=0.59$ ).

\section{Current wheeze in early childhood}

The prevalence of current wheeze diminished as the children aged, from $40 \%$ at age 1 year to $25 \%, 17 \%$ and $27 \%$ at ages 2,3 and 5 , respectively. However, the association between prenatal acetaminophen exposure and current wheeze strengthened as the children aged (figure $1 ; p=0.001$ ). There was no association between prenatal exposure to acetaminophen and visits to the emergency department for wheeze in the first 3 years of life or seroatopy at age $2-3$ years (data not shown).

\section{Effect modification by genotype}

Among the African-American and Dominican Republic children in this cohort, the frequencies of the GSTP1 minor allele (46\% vs $43 \%)$, deletions of GSTM1 (22\% vs $21 \%$ ) and deletions of GSTT1 (19\% vs $24 \%$ ) were similar, so the analyses of these interactions were not stratified by race/ethnicity. The distribution of GSTT2 alleles did differ by ethnicity and stratification by race/ethnicity limited statistical power to meaningfully test interactions with this gene polymorphism. There were no statistically significant associations between any of the three GST polymorphisms and either wheeze or seroatopy (table 3).

The magnitude of the association of prenatal acetaminophen exposure and current wheeze at age 5 years was modified by the presence of the minor GSTP1 allele (RERI 0.82; 95\% CI 0.20 to 1.44; $\mathrm{p}=0.009$ ) (table 3). Prenatal exposure to acetaminophen predicted current wheeze (RR 2.08; 95\% CI 1.34 to 3.23 ; $\mathrm{p}=0.001)$ and seroatopy (RR $1.96 ; 95 \%$ CI 1.36 to $2.84 ; \mathrm{p}<0.001)$ at age 5 years among children with at least one copy of the GSTP1 minor allele (AG, GG), but not among the children homozygous for the major allele (AA) (figure 2). Evidence for effect modification by gene variants in GSTT1 was also present (interaction with seroatopy $\mathrm{p}<0.001$ ). There were no statistically significant interactions with deletions in GSTM1.

\section{DISCUSSION}

Exposure to acetaminophen in the second and third trimesters of pregnancy predicted current wheeze at age 5 years in addition to other measures of asthma and seroatopy among African-American and Dominican Republic children living in an inner-city community with a high burden of asthma. The association of prenatal exposure to acetaminophen and current wheeze was modified by a common polymorphism in the GSTP gene, suggesting a glutathione-related mechanism.

Prenatal exposure to acetaminophen predicted current wheeze in children age 4-6years in earlier studies from the UK, Denmark and Spain ${ }^{5-7}$ and also predicted wheeze in the first year of life in one US study. ${ }^{29}$ The frequency of acetaminophen use during pregnancy and the magnitude of association in the UK study were similar to that in New York City. The prevalence of current wheeze at 6 years, however, was only $10 \%$ in the UK study, such that the attributable risk of acetaminophen was $1 \% .^{5}$ In contrast, the prevalence of current wheeze in the present study was $27 \%$, resulting in a population attributable risk from acetaminophen exposure assuming a causal relationship of $6 \%$.

The relevant timing of acetaminophen exposure during pregnancy is not clear, with the UK study and our study showing similar point estimates for risk with exposure early and late in pregnancy and the Danish study showing stronger associations with exposure early in pregnancy. ${ }^{6}$ Other differences include 
Table 2 Relative risk (RR) for wheeze and related outcomes at age 5 years with prenatal acetaminophen exposure $\left(n=297^{*}\right)$

\begin{tabular}{|c|c|c|c|c|c|c|}
\hline & & \multicolumn{4}{|c|}{ Reported days of acetaminophen use in pregnancy } & \multirow{2}{*}{$\begin{array}{l}p \text { Value } \\
\text { for trend }\end{array}$} \\
\hline & & $0(n=198)$ & $1(n=37)$ & $2-4(n=37)$ & $\geq 5(n=25)$ & \\
\hline \multirow[t]{2}{*}{ Current wheeze } & No (\%) & $41(21)$ & $11(30)$ & $14(38)$ & $13(52)$ & \multirow[t]{2}{*}{$<0.001$} \\
\hline & Crude RR $(95 \%$ Cl) & 1 (reference) & $1.44(0.82$ to 2.53$)$ & $1.83(1.11$ to 3.00$)$ & 2.51 (1.58 to 4.00$)$ & \\
\hline \multirow[t]{3}{*}{ Persistent wheeze $\neq$} & No (\%) & $34(17)$ & $9(24)$ & $12(32)$ & $9(36)$ & \multirow[t]{2}{*}{0.008} \\
\hline & Crude RR (95\% Cl) & 1 (reference) & $1.42(0.74$ to 2.70$)$ & $1.89(1.10$ to 3.30$)$ & 2.10 (1.14 to 3.84$)$ & \\
\hline & Adjusted RR (95\% Cl) & 1 (reference) & $1.29(0.72$ to 2.32$)$ & $1.94(1.22$ to 3.08$)$ & $1.90(1.15$ to 3.15$)$ & 0.009 \\
\hline \multirow{2}{*}{ Sleep disturbed by wheeze } & Crude RR $(95 \%$ Cl) & 1 (reference) & $0.71(0.27$ to 1.91$)$ & 1.78 (0.96 to 3.32$)$ & 2.38 (1.28 to 4.41$)$ & 0.005 \\
\hline & Adjusted RR (95\% Cl) & 1 (reference) & $0.48(0.14$ to 1.62$)$ & 2.05 (1.21 to 3.47 ) & 2.39 (1.31 to 4.34$)$ & 0.005 \\
\hline \multirow[t]{3}{*}{ Took medication for wheeze or difficulty breathing } & No (\%) & $46(23)$ & $13(35)$ & $15(41)$ & $13(52)$ & \multirow[t]{2}{*}{0.001} \\
\hline & Crude RR (95\% Cl) & 1 (reference) & 1.51 (0.91 to 2.51$)$ & 1.74 (1.10 to 2.78 ) & 2.24 (1.42 to 3.52 ) & \\
\hline & Adjusted RR $(95 \%$ Cl) & 1 (reference) & 1.24 (0.72 to 2.11$)$ & 1.97 (1.34 to 2.89 ) & 2.18 (1.40 to 3.42$)$ & $<0.001$ \\
\hline $\begin{array}{l}\text { Emergency department or emergency visit } \\
\text { to a physician for asthma, wheeze or other } \\
\text { breathing problem }\end{array}$ & No $(\%)$ & $31(16)$ & $10(27)$ & $12(32)$ & $7(28)$ & 0.034 \\
\hline Seroatopy§ & Adjusted RR $(95 \% \mathrm{Cl})$ & 1 (reference) & 1.21 (0.68 to 2.36$)$ & $1.32(0.81$ to 2.16$)$ & 1.99 (1.36 to 2.91$)$ & $<0.001$ \\
\hline
\end{tabular}

*The four mothers who reported acetaminophen use during pregnancy but did not report the number of days of use were excluded from this table.

†Relative risks were adjusted for sex, ethnicity, birth order, maternal asthma, maternal hardship, exposure to environmental tobacco smoke and postnatal acetaminophen use.

¥Wheeze was reported at least once between birth, age 3 years AND in the past 12 months at age 5 years.

$\S$ Child had specific IgE ( $>0.35 \mathrm{IU} / \mathrm{ml})$ to at least one allergen tested: dust mite (Dermatophagoides farinae), cockroach, mouse, cat or dog.

qThe number of children on whom $\lg E$ was measured was lower $(n=221)$ than those with symptom data at age 5.

effect modification by maternal asthma in the study from Spain, which we did find in our inner-city cohort. ${ }^{7}$ In contrast to the multicentre European study, we observed no association between postnatal acetaminophen use and wheeze. ${ }^{4}$

One reason for the larger potential impact of prenatal exposure to acetaminophen on current wheeze in this minority cohort may be differences in the GSTP1 minor allele frequency. Prenatal acetaminophen exposure was strongly associated with wheeze in participants with the GSTP1 minor allele, whereas no association was evident among those homozygous for the major allele. The minor allele frequency of GSTP1 was not reported in the predominantly Caucasian UK cohort; however, another study of Caucasians in the UK found a minor allele frequency of $32 \%{ }^{30}$ Similar frequencies have been observed in asthma studies from Taiwan (18\%) and Sweden (33\%) $)^{11} 12$ and among nonHispanic white subjects (32\%) in Southern California. ${ }^{10}$ In contrast, the observed minor allele frequency of $44 \%$ among African-Americans and Dominican Republic subjects in the current study was similar to that among African-Americans $(43 \%)$ and Hispanics (43\%) in Southern California. ${ }^{10}$

The mechanism of the association between acetaminophen and asthma has been hypothesised to involve the glutathione pathway which is downregulated following acetaminophen exposure and among subjects with asthma. ${ }^{31}$ GSTP catalyses the conjugation of reduced glutathione to a wide variety of substrates for detoxification and is responsible for $90 \%$ of the GST activity in the lungs. Also, a small portion of acetaminophen is converted to n-acetylbenzoquinonimine (NAPOI) by cytochrome p450, which can be cytotoxic. ${ }^{17}$ Shaheen et al have suggested the drug-induced upregulated action of NAPOI in the cells of the developing fetal lungs as a potential mechanism between prenatal acetaminophen and asthma. ${ }^{5}$ Furthermore, GSTP catalyses conjugation of NAPOI to glutathione at a rate faster than other GSTs. ${ }^{32}$ Since GSTP itself can be damaged NAPQI, pulmonary toxicity could occur as a result of direct activity by NAPOI or indirectly by decreased GSTP conjugation activity of other toxicants. ${ }^{17}$
The common polymorphism in GSTP (GSTP1 A105G) leads to expression of GSTPs with functionally different catalytic capacities. ${ }^{15} 33$ Recently, polymorphisms in GSTP1 have been reported to alter asthma and allergy susceptibility to diesel, ETS and ambient air pollution. ${ }^{9-13}$ GSTP is also involved in other pathways, including regulation of the stress-activated proteins c-Jun N-terminal kinases (JNK), which can activate transcription factors within the allergic pathway, potentially offering a connection between acetaminophen and atopy through GSTP. ${ }^{35}$ We observed an interaction between the GSTT deletion and acetaminophen on atopy but not wheeze, suggesting that the acetaminophen relationship with atopy and wheeze may act

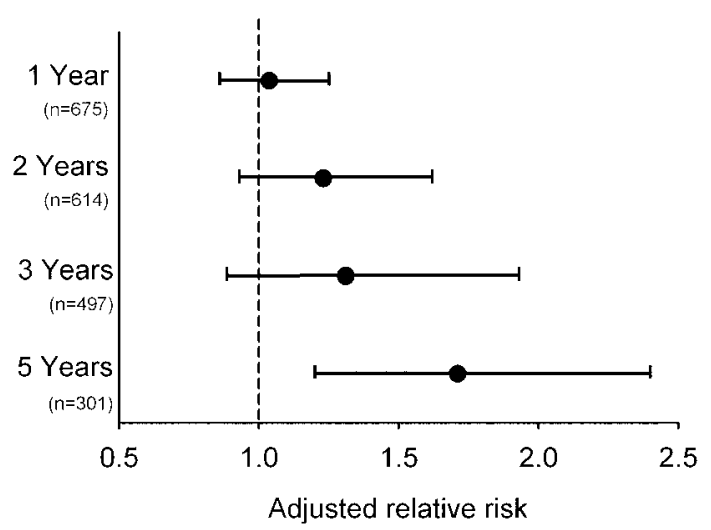

Figure 1 Adjusted relative risk for current wheeze (previous 12 months) with reported prenatal exposure to acetaminophen. Relative risks were adjusted for sex, race/ethnicity, birth order, maternal asthma, maternal hardship, exposure to environmental tobacco smoke and postnatal acetaminophen use. Bars represent $95 \%$ confidence intervals. A trend of increasing risk of wheeze with age $(p=0.001)$ was observed. When the analysis was restricted to the 301 children who had reached age 5 , the relative risks for wheeze at ages 1, 2 and 3 with prenatal acetaminophen exposure were 1.11 (95\% $\mathrm{Cl} 0.87$ to 1.42 ); 1.62 (1.10 to 2.37 ); and 1.39 (0.89 to 2.16$)$, respectively. 
Table 3 Risk for current wheeze and seroatopy by common GSTP1, GSTM1, GSTT1 polymorphisms $†$ and tests for additive interaction‡

\begin{tabular}{|c|c|c|c|c|c|c|}
\hline GSTP1 SNP & & $\mathrm{AA}$ & AG & GG & RERI $(95 \% \mathrm{CI}) \ddagger$ & $p$ Value \\
\hline \multirow{2}{*}{ Wheeze, n (\%) } & Overall & $25 / 87(29)$ & $39 / 157(25)$ & $16 / 53(30)$ & & 0.85 \\
\hline & Prenatal acetaminophen & $11 / 35(31)$ & $21 / 49(43)^{*}$ & $8 / 18(44)$ & $0.82(0.20$ to 1.4$)$ ब & 0.009 \\
\hline \multirow{2}{*}{ Seroatopy§, n (\%) } & No prenatal acetaminophen & $11 / 38(29)$ & $20 / 74(27)$ & $5 / 29(17)$ & & \\
\hline & Prenatal acetaminophen & $7 / 27(26)$ & $15 / 37(41)$ & $8 / 16(50)^{*}$ & 0.75 (0.065 to 1.4$) \Phi$ & 0.032 \\
\hline GSTT1 SNP & & Present & Null & & RERI $(95 \%$ Cl) & \\
\hline Wheeze, $n(\%)$ & Prenatal acetaminophen & $32 / 79(41)^{*}$ & $8 / 24(33)$ & & $-0.37(-1.8$ to 1.0$)$ & 0.60 \\
\hline \multirow[t]{3}{*}{ Seroatopy, n (\%) } & Overall & $57 / 177(32)$ & $10 / 45(22)$ & & & 0.19 \\
\hline & No prenatal acetaminophen & $29 / 114(25)$ & $8 / 28(29)$ & & & \\
\hline & Prenatal acetaminophen & $28 / 63(44)^{*}$ & $2 / 17(12)$ & & $0.98(0.50$ to 1.5$)$ & $<0.001$ \\
\hline GSTM1 SNP & & Present & Null & & RERI $(95 \% \mathrm{Cl})$ & \\
\hline Wheeze, $n(\%)$ & Overall & $64 / 228(28)$ & $15 / 61(25)$ & & & 0.59 \\
\hline Seroatopy, n (\%) & Prenatal acetaminophen & $23 / 61(38)^{*}$ & $6 / 18(33)$ & & $0.087(-0.71$ to 0.88$)$ & 0.83 \\
\hline
\end{tabular}

${ }^{*} \mathrm{p}<0.05$ For the difference in frequency of wheeze/seroatopy between children with and without prenatal acetaminophen exposure.

†Four common polymorphisms in glutathione S transferases were hypothesised to potentially modify the main effect of prenatal acetaminophen and wheeze. One SNP, GSTT2, was not similarly distributed among the Dominican Republic and African-American children and was therefore not tested in the interaction analysis. The remaining three GST SNPs were tested for interaction with the primary outcome (current wheeze) and one secondary outcome (seroatopy). The GSTP1 allele distribution met Hardy-Weinberg equilibrium criteria ( $\mathrm{p}>0.2$ for difference from expected). ¥The statistical significance of effect modification by genotype was assessed by estimating the relative excess risk due to interaction (RERI) and its $95 \%$ confidence interval as described by Hosmer and Lemeshow using the fully adjusted model. ${ }^{27} 28$

$\S$ Child had specific $\lg E(>0.35 \mathrm{IU} / \mathrm{ml})$ to at least one allergen tested: dust mite (Dermatophagoides farinae), cockroach, mouse, cat or dog.

बFor the GSTP1 interaction analysis, children with at least one copy of the minor allele (ie, AG or GG) were grouped and compared with those without (eg, AA) based on previous associations with asthma ${ }^{11}{ }^{13}$ and altered conjugation kinetics of toxicants related to asthma among individuals with this genotype. ${ }^{15}$

via the glutathione pathway, although not necessarily in identical ways. Our finding of a significant interaction between acetaminophen and GSTP1 offers the first evidence of a direct connection between acetaminophen exposure and asthma through the glutathione pathway.

We observed an association between prenatal exposure to acetaminophen and persistent but not transient wheeze. This, combined with our observation of increased risk for outcomes associated with more severe asthma (eg, emergency department visits for asthma) and atopy, suggests that prenatal exposure to acetaminophen increases the risk for phenotypes of asthma that are more likely to persist throughout childhood. ${ }^{21}$

The observational nature of this study means that the findings may be confounded by unmeasured or mismeasured covariates that cause asthma and were associated with prenatal acetaminophen exposure. However, we adjusted for the major known risk factors for paediatric asthma and the observed associations were not attenuated. A limitation was not ascertaining the reason for prenatal acetaminophen use, but the observed associations with headaches suggest pain management as likely. However, other host factors that caused mothers to take acetaminophen and also cause asthma may explain their association. Infection is one such potential confounder; however, we found no association between the reported use of antibiotics and acetaminophen, and adjustment for antibiotic use during pregnancy did not affect the results. An anxious personality also could have confounded the association; however, it is unlikely that this would explain the observed genetic interaction. Systematic reporting bias for both prenatal exposure to acetaminophen and wheeze at different ages seems unlikely given the prospective design of the study, the observed relationship of acetaminophen exposure with seroatopy and the effect modification by GSTP1. An additional limitation is the sample size and possibility of a false-positive result owing to multiple compari- sons. However, we specified one primary end point a priori (wheeze) and the statistical significance of the association between prenatal exposure to acetaminophen and this outcome was at the level of $p<0.001$. Our tests for interactions with genotype polymorphisms were limited to three functional gene polymorphisms related to a previously hypothesised pathway (glutathione), and these three were tested for interaction with one primary (wheeze) and one secondary (atopy) outcome. The interaction with GSTP1 and prenatal acetaminophen on wheeze

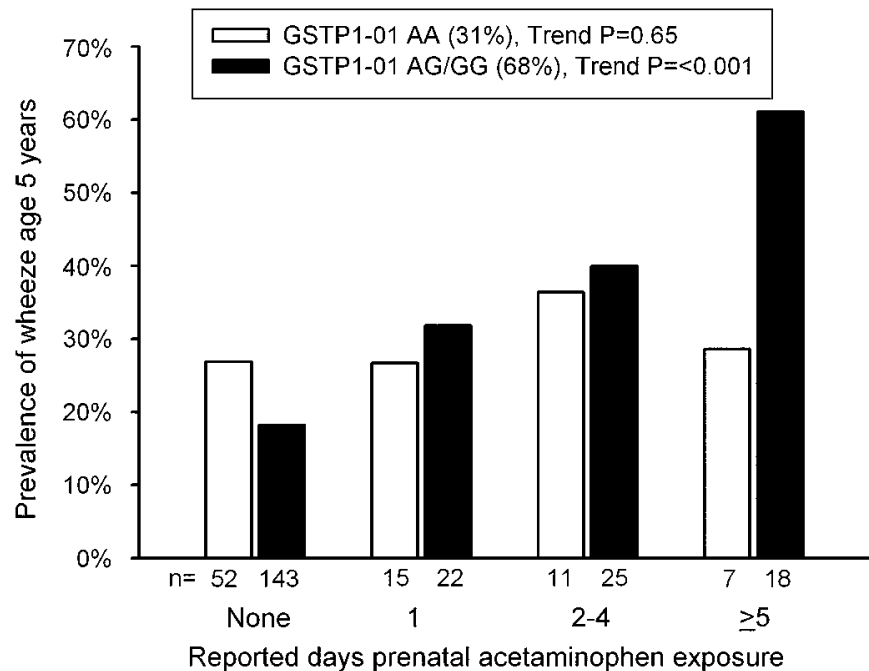

Figure 2 Association between days of acetaminophen use during pregnancy and current wheeze at age 5 years stratified by common polymorphism in the GSTP1 gene. The interaction between any prenatal acetaminophen use and the presence of a $G$ allele ( $A G$ or $G G$ ) in the GSTP1 (A105G) polymorphism on wheeze at age 5 was statistically significant $(p=0.009)$ in fully adjusted analyses. 
was statistically significant at the level of $p<0.01$. The $31 \%$ of the children who were lost to follow-up at age 5 years were more likely to be African-American and their mothers were more likely to complain of severe headaches and abdominal cramps in pregnancy, but there was no difference in reported prenatal acetaminophen use with these mothers and regression models were adjusted for race/ethnicity and complaints about headaches and pain. A further limitation of our study was the generalisability since mothers who smoked during pregnancy and those younger than 18 were excluded from the study.

In conclusion, prenatal acetaminophen exposure predicted asthma symptoms among African-American and Dominican Republic children-a relationship that was stronger among children with the common GSTP1 minor allele. These findings might provide an explanation for some of the increased asthma risk in minority communities and suggest caution in the use of acetaminophen in pregnancy.

Acknowledgements The authors would like to thank the participating mothers and children. This work would not have been possible without the hard work and dedication of the research workers and field technicians. The authors would also like to thank Drs Ruth Ottman and Erik Melén for their review of the manuscript.

Funding This study was supported by the National Institute of Environmental Health Sciences (NIEHS) (grant \#s P01 ES09600, 5 R01 ES08977, R01ES11158, P30 ES009089, P50ES015905), the U.S. Environmental Protection Agency (EPA) (grant \#s R827027, RD-832141), Bauman Family Foundation, Gladys and Roland Harriman Foundation, New York Community Trust, Educational Foundation of America, The New York Times Company Foundation, The Schmidt Family Foundation, The Johnson Family Foundation, Rockefeller Financial Services, Horace W Goldsmith Foundation, Beldon Fund, The John Merck Fund and V Kann Rasmussen Foundation. GLC received funding as a NIH National Center on Minority Health and Health Disparities fellow. RGB was supported by a Robert Wood Johnson Generalist Faculty Scholars Award. Other funders: $\mathrm{NIH}$.

\section{Competing interests None.}

Ethics approval The study was approved by Columbia University's Institutional Review Board.

Provenance and peer review Not commissioned; externally peer reviewed.

\section{REFERENCES}

1. Varner AE, Busse WW, Lemanske RF Jr. Hypothesis: decreased use of pediatric aspirin has contributed to the increasing prevalence of childhood asthma. Ann Allergy Asthma Immunol 1998;81:347-51.

2. Werler MM, Mitchell AA, Hernandez-Diaz S, et al. Use of over-the-counter medications during pregnancy. Am J Obstet Gynecol 2005;193(3 Pt 1):771-7.

3. Moorman JE, Rudd RA, Johnson CA, et al. National surveillance for asthma-United States, 1980-2004. MMWR Surveill Summ 2007:56:1-54.

4. Beasley R, Clayton T, Crane J, et al. Association between paracetamol use in infancy and childhood, and risk of asthma, rhinoconjunctivitis, and eczema in children aged 6-7 years: analysis from Phase Three of the ISAAC programme. Lancet 2008;372:1039-48.

5. Shaheen SO, Newson RB, Henderson AJ, et al. Prenatal paracetamol exposure and risk of asthma and elevated immunoglobulin $\mathrm{E}$ in childhood. Clin Exp Allergy 2005;35:18-25.

6. Rebordosa C, Kogevinas M, Sorensen HT, et al. Pre-natal exposure to paracetamol and risk of wheezing and asthma in children: a birth cohort study. Int J Epidemiol 2008;37:583-90.

7. Garcia-Marcos L, Sanchez-Solis M, Perez-Fernandez V, et al. Is the effect of prenatal paracetamol exposure on wheezing in preschool children modified by asthma in the mother? Int Arch Allergy Immunol 2009:149:33-7.

8. Busse WW, Mitchell $\mathrm{H}$. Addressing issues of asthma in inner-city children. J Allergy Clin Immunol 2007;119:43-9.

9. Gilliland FD, Li YF, Saxon A, et al. Effect of glutathione-S-transferase M1 and P1 genotypes on xenobiotic enhancement of allergic responses: randomised, placebocontrolled crossover study. Lancet 2004;363:119-25.
10. Gilliland FD, Gauderman WJ, Vora H, et al. Effects of glutathione-S-transferase M1, T1, and P1 on childhood lung function growth. Am J Respir Crit Care Med 2002:166:710-6.

11. Lee YL, Lee YC, Guo YL. Associations of glutathione S-transferase P1, M1, and environmental tobacco smoke with wheezing illness in school children. Allergy 2007:62:641-7.

12. Melen E, Nyberg F, Lindgren CM, et al. Interactions between glutathione S-transferase P1, tumor necrosis factor, and traffic-related air pollution for development of childhood allergic disease. Environ Health Perspect 2008; 116:1077-84.

13. Schroer KT, Biagini Myers JM, Ryan PH, et al. Associations between multiple environmental exposures and glutathione S-transferase P1 on persistent wheezing in a birth cohort. J Pediatr 2008.

14. Romieu I, Ramirez-Aguilar M, Sienra-Monge JJ, et al. GSTM1 and GSTP1 and respiratory health in asthmatic children exposed to ozone. Eur Respir $\mathrm{J}$ 2006;28:953-9.

15. Watson MA, Stewart RK, Smith GB, et al. Human glutathione S-transferase P1 polymorphisms: relationship to lung tissue enzyme activity and population frequency distribution. Carcinogenesis 1998;19:275-80.

16. Henderson CJ, Wolf CR, Kitteringham N, et al. Increased resistance to acetaminophen hepatotoxicity in mice lacking glutathione S-transferase Pi. Proc Nat/ Acad Sci U S A 2000;97:12741-5.

17. Jenkins RE, Kitteringham NR, Goldring CE, et al. Glutathione-S-transferase P1 as a model protein for the characterisation of chemically reactive metabolites. Proteomics 2008:8:301-15.

18. Miller RL, Chew GL, Bell CA, et al. Prenatal exposure, maternal sensitization, and sensitization in utero to indoor allergens in an inner-city cohort. Am J Respir Crit Care Med 2001:164:995-1001.

19. Perzanowski MS, Chew GL, Divjan A, et al. Cat ownership is a risk factor for the development of anti-cat $\lg E$ but not current wheeze at age 5 years in an inner-city cohort. J Allergy Clin Immunol 2008;121:1047-52.

20. Worldwide variation in prevalence of symptoms of asthma, allergic rhinoconjunctivitis, and atopic eczema: ISAAC. The International Study of Asthma and Allergies in Childhood (ISAAC) Steering Committee. Lancet 1998;351:1225-32.

21. Martinez FD, Wright AL, Taussig LM, et al. Asthma and wheezing in the first six years of life. The Group Health Medical Associates. N Engl J Med 1995; 332:133-8.

22. Miller RL, Garfinkel R, Horton $M$, et al. Polycyclic aromatic hydrocarbons, environmental tobacco smoke, and respiratory symptoms in an inner-city birth cohort. Chest 2004;126:1071-8.

23. Perzanowski MS, Canfield SM, Chew GL, et al. Birth order, atopy, and symptoms of allergy and asthma among inner-city children attending Head Start in New York City. Clin Exp Allergy 2008;38:968-76.

24. Rauh VA, Whyatt RM, Garfinkel R, et al. Developmental effects of exposure to environmental tobacco smoke and material hardship among inner-city children. Neurotoxicol Teratol 2004;26:373-85.

25. Arand $\mathbf{M}$, Muhlbauer R, Hengstler J, et al. A multiplex polymerase chain reaction protocol for the simultaneous analysis of the glutathione S-transferase GSTM1 and GST1 polymorphisms. Anal Biochem 1996;236:184-6.

26. Wacholder $\mathbf{S}$. Binomial regression in GLIM: estimating risk ratios and risk differences Am J Epidemiol 1986;123:174-84.

27. Andersson T, Alfredsson L, Kallberg $\mathrm{H}$, et al. Calculating measures of biological interaction. Eur J Epidemiol 2005;20:575-9.

28. Hosmer DW, Lemeshow S. Confidence interval estimation of interaction. Epidemiology 1992;3:452-6.

29. Persky V, Piorkowski J, Hernandez E, et al. Prenatal exposure to acetaminophen and respiratory symptoms in the first year of life. Ann Allergy Asthma Immunol 2008; 101:271-8.

30. Carroll WD, Lenney W, Jones PW, et al. Effects of glutathione S-transferase M1, $\mathrm{T} 1$ and $\mathrm{P} 1$ on lung function in asthmatic families. Clin Exp Allergy 2005;35 1155-61.

31. Eneli I, Sadri K, Camargo CA, et al. Acetaminophen and the risk of asthma: the epidemiologic and pathophysiologic evidence. Chest 2005;127:604-12.

32. Coles B, Wilson I, Wardman P, et al. The spontaneous and enzymatic reaction of $\mathrm{N}$-acetyl-p-benzoquinonimine with glutathione: a stopped-flow kinetic study. Arch Biochem Biophys 1988;264:253-60.

33. Fryer AA, Hume R, Strange RC. The development of glutathione S-transferase and glutathione peroxidase activities in human lung. Biochim Biophys Acta 1986:883:448-53.

34. Sundberg K, Johansson AS, Stenberg G, et al. Differences in the catalytic efficiencies of allelic variants of glutathione transferase P1-1 towards carcinogenic diol epoxides of polycyclic aromatic hydrocarbons. Carcinogenesis 1998;19:433-6.

35. Adler V, Yin Z, Fuchs SY, et al. Regulation of JNK signaling by GSTp. Embo J 1999:18:1321-34. 